\title{
System Frequency as Information Carrier in AC Power Systems
}

\author{
Douglass, Philip James; Heussen, Kai; You, Shi; Gehrke, Oliver; Østergaard, Jacob
}

\section{Published in:}

IEEE Transactions on Power Delivery

Link to article, DOI:

10.1109/tpwrd.2014.2335694

Publication date:

2015

Document Version

Peer reviewed version

Link back to DTU Orbit

Citation (APA):

Douglass, P. J., Heussen, K., You, S., Gehrke, O., \& Østergaard, J. (2015). System Frequency as Information Carrier in AC Power Systems. IEEE Transactions on Power Delivery, 30(2), 773-782.

https://doi.org/10.1109/tpwrd.2014.2335694

\section{General rights}

Copyright and moral rights for the publications made accessible in the public portal are retained by the authors and/or other copyright owners and it is a condition of accessing publications that users recognise and abide by the legal requirements associated with these rights.

- Users may download and print one copy of any publication from the public portal for the purpose of private study or research.

- You may not further distribute the material or use it for any profit-making activity or commercial gain

- You may freely distribute the URL identifying the publication in the public portal 


\title{
System Frequency as Information Carrier in AC Power Systems
}

\author{
Philip J. Douglass Member, IEEE, Kai Heussen Member, IEEE, Shi You Member, IEEE, Oliver Gehrke, \\ Jacob Østergaard Senior Member, IEEE
}

\begin{abstract}
Power generators contain control systems able to regulate system frequency, but the frequency setpoint values are only rarely modified from nominal values. This paper describes design considerations for a communication system from generators to frequency sensitive distributed energy resources (FS-DER) using changes to frequency setpoint values of generators. Signaling discrete system states by generating off-nominal system frequency values can be used as a novel narrowband unidirectional broadcast communications channel. This paper describes two protocols for utilizing off-nominal frequencies to carry information: First, a protocol for dispatching blocks of FSDER that is suitable for systems restricted to relatively slow rates of change of frequency (ROCOF). Second, for systems that allow higher ROCOF values, the feasibility of using power generation resources as a power line communication transmitter is shown. Data from an operating islanded power system with diesel generators is analyzed to demonstrate the feasibility of the proposed communication system in systems fed by rotating machines. The feasibility of the proposed communication system in systems fed by voltage source inverters is shown with laboratory tests of a $20 \mathrm{kVA}$ inverter. The inverter was found to have a maximum ROCOF of $2.2 \mathrm{~Hz} / \mathrm{s}$, sufficient to enable its use as a power line communication transmitter.
\end{abstract}

Index Terms-Power Frequency Communication; Ultra Narrow Band; Energy Management; Load Management; Voltage Source Inverters; Distributed Energy Resources; ROCOF.

\section{INTRODUCTION}

$\mathbf{T}$ RANSITIONING electric energy systems to renewable energy sources (RES) will increase the sustainability as well as the complexity of the power system. The low power density of RES favors distributed generation (DG), and as a result, the number of entities actively participating in maintaining system stability will increase greatly. The fluctuating production profile of RES and scarcity of large-scale electric energy storage motivates the creation of energy management systems that include loads.

While the availability of low-cost microcontrollers allows software control of even the smallest loads, creating a communications network that reaches out to many separate units is a costly endeavor. Real-time constraints on the control of power systems impose additional costs on communication systems. And where such a communication system has been established, maintaining the $\mathrm{N}-1$ protection criteria requires that failure of the communication system does not compromise system security. Therefore a robust fallback state must be defined.

The authors are with Center for Electric Power and Energy, Technical University of Denmark, Elektrovej-Building 325, 2800 Kgs. Lyngby, Denmark \{pjdo, kh, sy, olge, joe\}@elektro.dtu.dk.
The physical characteristics of the voltage waveform (AC frequency and RMS voltage) can be measured at the point of energy consumption, and can reveal valuable information about the state of the power system. Utilizing local measurements for autonomous control of distributed energy resources (DER) was first proposed in [1]. The physical characteristics of the voltage waveform are subject to disturbances, but to the extent that system operators can maintain the parameters within known intervals, the parameters can be used for communication.

Power line communication has been in use since the pioneering days of public electric energy systems. Using the AC system frequency $(50 \mathrm{~Hz}$ or $60 \mathrm{~Hz})$ as a carrier for frequency modulation, called power frequency communication (PFC), was first introduced with the TWACS communication system [2]. The TWACS transmitters described in [2], [3] are intended for modifying the frequency of a single radial feeder in a large synchronous area. This paper investigates a general framework for using $\mathrm{AC}$ system frequency for transmitting information which differs from TWACS in the following ways: the information reaches an entire synchronous area; it can utilize more of the available bandwidth; it can operate with or without time-dependent decoding; and it utilizes energy sources as transmitters. The motivation for this framework is to create energy management systems with lower costs and higher reliability relative to existing alternatives by reducing the number of system components.

An overview of the communication system model, adapted from the authors' previous work in [4], is shown in Fig. 1. Frequency sensitive distributed energy resources (FS-DER) and conventional passive loads are connected to a controllable generator through a power grid. A series of symbols $\left(S_{k}, S_{k+1}, \ldots\right)$ are mapped to frequency set point changes that are given to the generator. The generator changes the system frequency, and these changes are measured by FS-DER, which is able to reconstruct the original series of symbols.

This paper describes two protocols for encoding information with system frequency. The first protocol dispatches FS-DER by transmitting the value of a single discrete parameter and is suitable for large power systems with slow frequency dynamics. The second protocol creates a general purpose PFC channel in systems with fast frequency dynamics.

The remainder of the paper is structured as follows: Section II gives background in frequency control of power systems. Section III describes existing PFC systems. Section IV describes the general methods for deriving constraints for the communication system that are applied in Sections V \& VI to 


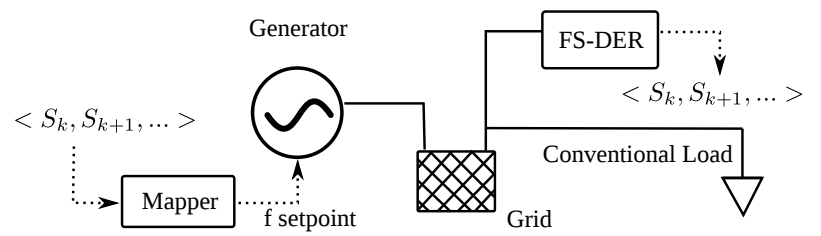

Figure 1. Communication system model with solid lines representing electrical energy transmission paths and dashed lines representing logical signaling paths. Symbols $\left(S_{k}, S_{k+1}, \ldots\right)$ are encoded into system frequency values which are detected by FS-DER.

FS-DER dispatch and PFC transmission respectively. Section VII shows the feasibility of the proposed communication schemes with case studies of two types of islanded power systems: a diesel dominated system, and an inverter dominated system. Section IX is a discussion, and Section X concludes the paper.

\section{Power System Frequency Control}

Power systems must keep system frequency within a narrow range, and frequency stability can be a limiting factor for increasing penetrations of fluctuating RES [5].

\section{A. Standards}

The EN 50160 standard [6] used in Europe specifies the physical characteristics of electric energy delivered to customers. A large interconnected power system is required to deliver power with a frequency specified as:

$50 \mathrm{~Hz} \pm 1 \%$ for $99.5 \%$ of the year

$50 \mathrm{~Hz}+4 \%,-6 \%$ for $100 \%$ of the time.

Small island power systems have a wider tolerance and must be designed for frequency constraints:

$50 \mathrm{~Hz} \pm 2 \%$ for $95 \%$ of a week

$50 \mathrm{~Hz} \pm 15 \%$ for $100 \%$ of the time.

Proposed grid codes from ENTSO-E [7] relax the constraints on system frequency for large systems, allowing unlimited operation in the range $50 \mathrm{~Hz} \pm 2 \%$. The proposed grid codes require generators to remain online at all times when the rate of change of frequency (ROCOF) is less than $2 \mathrm{~Hz} / \mathrm{s}$.

\section{B. Synchronous Machine Dominated Systems}

In a system with synchronous generators, system frequency is a noisy signal. Disturbances to system frequency are caused by imbalance between mechanical power provided to rotating generators $\Delta P_{\mathrm{g}}$ [p.u.] and electric power withdrawn by loads $\Delta P_{1}$ [p.u.]. In a single-machine system the ROCOF is given by the swing equation [8]:

$$
R O C O F=\Delta \dot{f}=\frac{f_{\mathrm{o}}}{2 H}\left(\Delta P_{\mathrm{g}}-\Delta P_{\mathrm{l}}\right)
$$

where $f[\mathrm{~Hz}]$ is the system frequency, $f_{\mathrm{o}}[\mathrm{Hz}]$ is the nominal frequency and $H$ [s] is the system inertia. When loads are uncontrollable, controlling generator power is the only way to maintain power balance.
For systems with multiple generators, Eq. (1) can be used to estimate the average system frequency by creating a lumped model of the system inertia:

$$
H=\sum_{i \in N} H_{i}
$$

where $H_{i}$ is the inertia contribution of each of $i$ generators in the set $N$. Power production $\left(\Delta P_{\mathrm{g}}\right)$ can be similarly aggregated.

In a multi-machine system, the frequencies observed at various busses are not uniform on very short time scales (from 10s of cycles to seconds) [9], a phenomena the lumped model can not describe. The effect of this short-term frequency variation is negligible in the proposed communication system because of a low-pass filter of frequency measurements in the FS-DER.

The response of the system to transient imbalances is determined by the magnitude of the power imbalance, the system's inertia and time constants on ramping up (or down) the primary and secondary frequency controlled reserves. During large imbalances, load shedding, or other special protection schemes are triggered and modify the system response. In the event of a disturbance, primary frequency regulation acts to stabilize the frequency on short time scales (seconds to minutes) by reestablishing the balance between generation and load. Primary frequency regulation is done by a $(P-f)$ droop response which makes generator power inversely proportional to system frequency. The droop response results in a steady state frequency deviation. To restore system frequency to the setpoint frequency, a slower secondary control loop responds to the integral of the frequency deviation.

Large synchronous generators are characterized by relatively slow response times to operating point changes. The spinning mass of the rotor contributes inertia to the system ( $H$ in eq. (1)), limiting the ROCOF during imbalances. Motors also contribute to system inertia, but relative to the rated power they contribute less than half the inertia of generators [10].

To dimension primary frequency regulation resources system operators perform simulation studies to model the response of the system to worst-case contingencies. These studies determine the minimum frequency (nadir) after the largest expected fault. Secondary frequency regulation resources are dimensioned to give an acceptable probability density of system frequency values. Power systems with high portions of fluctuating RES will not need fundamental changes to their primary frequency regulation resources [11], but secondary frequency regulation resources will be called upon to compensate for RES fluctuations [12]. Because the worst-case scenarios my depend on rare system configurations, significant benefits can be obtained from dynamically provisioning frequency regulation resources as proposed in [13].

In addition to limits on system frequency, ROCOF must also be kept within well defined constraints. Events that provoke high ROCOF values place stress on synchronous machines, and ROCOF sensitive relays are part of generator protection schemes. Grid-connected DG will often have ROCOF relays to prevent unintended island operation [14]. Today, interventions 


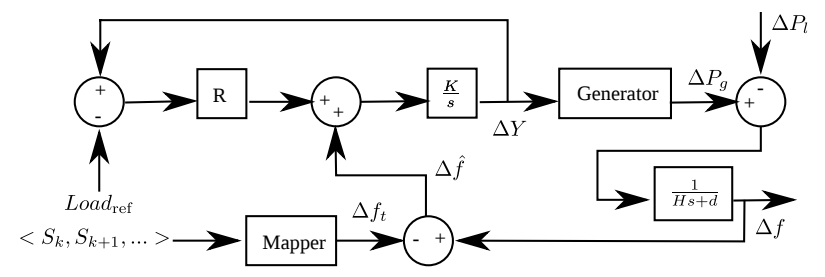

Figure 2. Simplified block diagram of a droop controlled generator used as a transmitter of information, adapted from [8]. A series of symbols $\left.<S_{k}, S_{k+1}, \ldots\right\rangle$ is encoded to frequency setpoint values $\Delta f_{t}$ by a simple 1-to-1 mapping and then given to the standard droop controller. The system's inertia $H$ and load damping factor $d$ determine how quickly a power imbalance $\left(\Delta P_{\mathrm{g}}-\Delta P_{\mathrm{l}}\right)$ changes system frequency $\Delta f$.

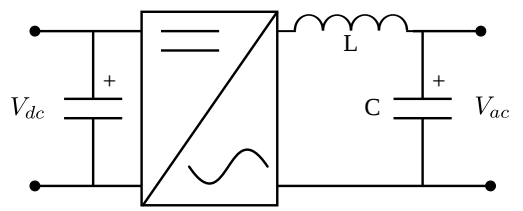

Figure 3. Inverter with LC output filter functions to convert DC primary energy source into AC.

are not generally needed to prevent the ROCOF from exceeding this requirement, but in synchronous areas with a growing penetration of non-synchronous RES generation, inertia will decline, and ROCOF will increase, to a level where mitigation efforts are needed [15].

When modeling the use of synchronous machines as transmitters of information, a generic generator is considered which covers all generation resources. Fig. 2 shows a block diagram of a conventional droop controlled generator whose speed governor is modulated with a stream of input symbols. The symbols are mapped to frequency setpoints using a lookup table. Decomposing the generator block in Fig. 2 of a rotating machine would reveal a transfer function from fuel delivery to turbine torque to electric power and the time delays involved in all sub-processes. Secondary frequency control is accomplished by changing $\operatorname{Load}_{\text {ref }}[\mathrm{MW}]$ values.

In large interconnected systems generator frequency setpoints are occasionally set to off-nominal values to correct deviations between electrical time and UTC. In the continental European power system, frequency setpoints are adjusted to $50.01 \mathrm{~Hz}$ or $49.99 \mathrm{~Hz}$ for up to 24 hours [16].

\section{Inverter Dominated Systems}

Inverters take a DC power source and apply power electronics to produce an $\mathrm{AC}$ sine wave with low distortion. The architecture of a generic inverter is shown in Fig. 3. They are classified as either voltage source inverters (VSI) or current source inverters (CSI). A system without rotating generators needs at least one VSI to produce a reference frequency to which CSI can synchronize [17]. Synchronous areas dominated by VSI can be found in any island where the primary power sources or transmission links are based on DC technology.

When energy sources are connected to an AC power system through VSIs, the system frequency is controlled by embedded real-time software, without physical constraints tied to the swing equation, eq. (1). In [18], a synchronous system was dubbed an "empty network" if all power sources and loads lacked inertia. In practical power systems fed only by inverters, physical inertia will be provided only by motors. If all rotating machines in a synchronous area are motors, or generators with constant power output, there will be no disturbances to the system frequency generated by the VSI, and frequency will be seen as constant on all busses.

In the context of using system frequency to carry information by changing frequency setpoint values, a low amount of physical inertia is an advantage because it allows rapid changes in system frequency (high ROCOF). For a VSI, the parameters of the generator block in Fig. 2 will have time delays on the order of the input sampling rate, well under $20 \mathrm{~ms}$. The short time delays enable VSIs to implement virtual inertia to mimic the behavior of synchronous machines.

\section{FS-DER for Primary Frequency Regulation}

Previous work on FS-DER has focused on using flexible loads to supplement large generators performing primary frequency regulation [19]-[21]. The availability of low-cost microelectronics to accurately measure system frequency has made it feasible for loads as small as household appliances to act as FS-DER for primary frequency regulation. Ongoing efforts to draft transnational grid codes in Europe cite the value of primary frequency regulation services from FS-DER to justify mandating their deployment on thermostat controlled loads [22].

A frequency sensitive load controller programmed for primary frequency regulation will shift the phase of the load duty cycle to defer energy consumption away from periods when the grid is overloaded, as indicated by a low system frequency.

The FS-DER algorithm for discrete relay-based devices described in [23] has a frequency threshold $f_{\text {off }}[\mathrm{Hz}]$ below which the load is disconnected. Loads are reconnected when system frequency is above a higher threshold frequency $f_{\text {on- }}$ $[\mathrm{Hz}]$, creating a simple hysteresis. A block diagram of the FSDER controller is shown in Fig. 4. Frequency measurements $f[k][\mathrm{Hz}]$ are sent through a low pass filter to produce $\hat{f}[k]$ $[\mathrm{Hz}]$ before comparing the value to preset thresholds. This filtering reduces the effects of noise in the frequency signal, and reduces the deviation between the measured frequency and the system average frequency.

The algorithm in [23] can be generalized to more complex DER that have more than two states of operation or to aggregated blocks of DER. The controller will transition to state $S$ at time $k$ when the measured and filtered system frequency $\hat{f}[k]$ enters the decision region given by:

$$
f_{S-} \leq \hat{f}[k] \leq f_{S+}
$$

Filtered system frequency $\hat{f}[k]$ must be between the lower frequency threshold $f_{S-}[\mathrm{Hz}]$ and the upper frequency threshold $f_{S+}[\mathrm{Hz}]$ to trigger a state change.

Some processes, like refrigerator compressors, have timing constraints on how quickly they can start-up and shutdown. When these devices are used as FS-DER and system frequency 


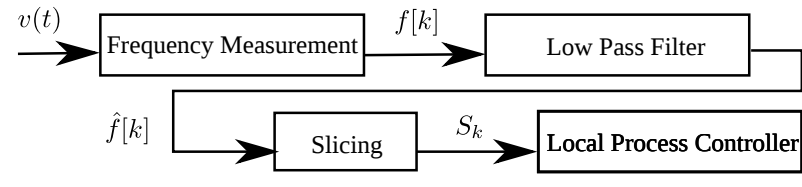

Figure 4. Block diagram of FS-DER controller. In time period $k$, the frequency $f[k]$ of the incoming voltage waveform $v(t)$ is filtered and then compared to pre-set threshold values (slicing), resulting in state $S_{k}$ which is given as input to the controller of the local energy conversion process.

fluctuates between the switching thresholds, the timing constraints take precedence over the frequency response. Therefore a reliable response from these FS-DER is only realized when they infrequently change state. To address this, section $\mathrm{V}$ describes how to design FS-DER thresholds and frequency setpoints to achieve a pre-determined probability of changing state.

\section{Power Frequency Communication (PFC)}

Standard frequency band allocation of the power line media gives utilities access to the lowest frequencies [24], and low bandwidth communication systems operating below the standard frequency range operate at speeds as low as $0.001 \mathrm{bps}$ [25]. Conventional power line communication superimposes high frequency components onto the fundamental energy carrying voltage waveform, but higher frequencies have greater signal attenuation per unit distance. In contrast, power frequency communication (PFC), which uses the energy carrying voltage waveform itself as a carrier for frequency modulation, is able to distribute signals without limitations on distance.

\section{A. Two Way Automatic Communication System (TWACS)}

TWACS is the an implementation of PFC currently utilized for remote meter reading, distribution automation, and outage management [26]. TWACS can modulate system frequency on a single radial feeder by injecting current to distort the downstream voltage waveform, thus modifying timing of the voltage zero-crossing. Because the AC voltage signal must be continuous phase, frequency shift keying is used to encode symbols. TWACS encodes up to two data bits with $1 \mathrm{AC}$ cycle, giving a data rate of $100 \mathrm{bps}$ [2].

A drawback of the original TWACS frequency modulation technique [3] is that the transmitting device introduces reactive power losses. The advent of synchronous areas dominated by inverter connected generation introduces the possibility of modulating system frequency at the power source at minimal marginal cost.

A TWACS receiver calculates system frequency by measuring the time elapsed between the zero-crossings, the same frequency measurement method as a FS-DER controller [27].

\section{B. Frequency Modulation in Inverter Dominated Micro-Grids}

In isolated micro-grids, VSIs available today can dispatch DG by changing the system frequency to off-nominal values. This feature relies on DG compliance with grid codes for large synchronous areas [28] that require CSIs to implement a $(P-f)$ droop when system frequency is above a critical value (i.e. $50.2 \mathrm{~Hz}$ ), thus providing emergency down regulation reserves. Modulating AC system frequency is used by VSI battery controllers to indicate battery charge status and to curtail production in islanded microgrid systems in the event that production exceeds load and energy storage devices are full [17], [29], [30].

These products demonstrate that VSIs can reliably produce off-nominal AC frequencies to communicate to DG, and they show that the normal operation of loads are not adversely effected by off-nominal frequencies. The protocol described in Section V modulates frequency, but unlike existing VSIs, does so in discrete domains. The resulting signal is used by all FS-DER, including both DG and loads.

\section{Constraints on Frequency Modulation}

This section describes methods for determining the constraints on system frequency setpoints. First, the limits to the range of frequency setpoint values (the bandwidth) are found. Second, limits to the rate of change of symbols (the baud rate) are found.

\section{A. Range of Frequency Setpoints}

To maintain system integrity, the minimum frequency after a large disturbance must be above the trip thresholds of under-frequency protection relays. If the maximum frequency deviation after a disturbance in the negative/positive direction is given as $\Delta f_{\max -} / \Delta f_{\max }[\mathrm{Hz}]$, and the technical minimum/maximum frequencies are $f_{\text {limit- }} / f_{\text {limit+ }}[\mathrm{Hz}]$, the system operator can choose frequency setpoints $f_{\mathrm{s}}[\mathrm{Hz}]$ within the interval $F_{\mathrm{s}, \text { dist }}$ given by:

$$
F_{\mathrm{s}, \text { dist }}=\left[f_{\text {limit- }}+\Delta f_{\text {max- }}, f_{\text {limit }}-\Delta f_{\text {max }+}\right] .
$$

The standards referenced in Section II-A require the system frequency to reside within a given interval $\mathrm{F}_{\mathrm{ok}}=\left[f_{\min }, f_{\max }\right]$ with a given likelihood $P_{\mathrm{ok}}$. This can be formulated as a constraint on the probability $\mathrm{P}$ of a frequency $f$ being observed within $\mathrm{F}_{\mathrm{ok}}$ during operation with frequency setpoint $f_{\mathrm{s}}$ :

$$
\mathrm{P}\left(f \in \mathrm{F}_{o k} ; f_{\mathrm{s}}\right) \geq \mathrm{P}_{\mathrm{ok}} .
$$

Consider the known probability density function $p\left(f ; f_{\mathrm{o}}\right)$ for a system operating with given frequency setpoint $f_{\mathrm{o}}$. We shall assume that small frequency offsets $\delta$ to $f_{\mathrm{o}}$ do not to change the shape of the probability density function (PDF):

$$
\mathrm{P}\left(f \in[a, b] ; f_{\mathrm{o}}\right)=\mathrm{P}\left(f \in[a+\delta, b+\delta] ;\left(f_{\mathrm{o}}+\delta\right)\right) .
$$

The set of permissible frequency setpoints $F_{\mathrm{s}, \mathrm{ok}}$ which satisfy eq. (4) is found by finding a frequency range where the probability of residing in $F^{\prime}$ during operation with frequency setpoint $f_{\mathrm{o}}$ is exactly $\mathrm{P}_{\mathrm{ok}}$ :

$$
\mathrm{P}\left(f \in F^{\prime} ; f_{\mathrm{o}}\right)=\mathrm{P}_{\mathrm{ok}} .
$$

The set $F^{\prime}$ is defined in the following expression:

$$
F^{\prime}=\left[f_{\mathrm{o}}-\Delta f_{\min }^{\prime}, f_{\mathrm{o}}+\Delta f_{\text {max }}^{\prime}\right] \subset \mathrm{F}_{\mathrm{ok}} .
$$

The parameters $\Delta f_{\min }^{\prime}, \Delta f_{\max }^{\prime}$ in eq. (7) can be derived by integrating across selected regions of the system frequency 


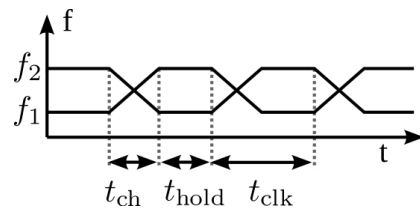

Figure 5. Timing diagram of PFC protocols. The time to change between symbols is $t_{\mathrm{ch}}$. The hold time $t_{\text {hold }}$ can be small.

PDF, see Section IV-C. The parameters become the margins that the operator must maintain between $f_{\min }, f_{\max }$ and $f_{s}$. As a result, the interval of $F_{\mathrm{s}, \mathrm{ok}}$ is given by:

$$
F_{\mathrm{s}, \mathrm{ok}}=\left[f_{\min }+\Delta f_{\min }^{\prime}, f_{\max }-\Delta f_{\max }^{\prime}\right] \text {. }
$$

The intersection of sets $F_{\mathrm{s} \text {,dist }}$ and $F_{\mathrm{s} \text {,ok }}$ gives the complete range of valid frequency setpoints $F_{\mathrm{s}}$, resulting in an available bandwidth, $f_{\mathrm{w}}[\mathrm{Hz}]$ :

$$
\begin{gathered}
F_{\mathrm{s}}=F_{\mathrm{s}, \text { dist }} \cap F_{\mathrm{s}, \mathrm{ok}} \\
f_{\mathrm{w}}=\max \left(F_{\mathrm{s}}\right)-\min \left(F_{\mathrm{s}}\right) .
\end{gathered}
$$

\section{B. Transmission Rate and ROCOF Constraints}

A system operator who chooses to vary system frequency setpoints will have to maintain ROCOF constraints during the transition from one frequency setpoint to another. A ROCOF budget will subtract the maximum ROCOF seen during a contingency $R O C O F_{\max }[\mathrm{Hz} / \mathrm{s}]$ from the technical limits $R O C O F_{\text {limit }}[\mathrm{Hz} / \mathrm{s}]$, and the resulting difference will give the budget for additional $R O C O F_{\mathrm{pfc}}[\mathrm{Hz} / \mathrm{s}]$ introduced when changing between frequency setpoints:

$$
R O C O F_{\mathrm{pfc}}=R O C O F_{\text {limit }}-R O C O F_{\max } .
$$

In systems with rotating machines $R O C O F_{\mathrm{pfc}}$ may also be limited by the power available from frequency regulation resources $P_{\text {reg }}$ [p.u.] relative to the system inertia, given by substituting $P_{\text {reg }}$ for the power imbalance in eq. (1). VSIs may have software limits on the maximum ROCOF.

A timing diagram showing the transition between frequency setpoints is shown in Fig. 5. The hold time $t_{\text {hold }}[\mathrm{s}]$ depends on the length of low pass filtering on the receiver, desired error rate, the size of the decision region and, where applicable, the accuracy of clock synchronization between sender and receiver.

The time taken for the system frequency to rise/fall $t_{\mathrm{ch}}$ [s] is a function of the spacing between frequency setpoint $f_{S}$ and its neighbor $f_{(S-1)}, \Delta f_{\mathrm{sp}}=f_{S}-f_{(S-1)}$, and the $R O C O F_{\mathrm{pfc}}$ :

$$
t_{\mathrm{ch}} \geq \frac{\Delta f_{\mathrm{sp}}}{R O C O F_{\mathrm{pfc}}} .
$$

The sum of the rise/fall time and hold time give the total time to transmit one symbol $t_{\mathrm{clk}}[\mathrm{s}]$ :

$$
t_{\mathrm{clk}}=t_{\mathrm{ch}}+t_{\mathrm{hold}}
$$

\section{System Frequency Probability Density Function}

The methods described in this paper assume the PDF of system frequency is known. The PDF can be determined empirically, as in [31], or by analysis. Analytically, the system frequency is a stochastic process with two regimes. During normal operation the values are close to $f_{s}$ and the PDF conforms well to a Gaussian distribution. This regime is characterized by the random (de-)activation of loads and efforts of the primary and secondary frequency controllers on generators to restore the frequency to the setpoint value. However, extreme frequency excursions as a result of faults occur more often than a Gaussian distribution predicts, resulting in a "fat tail" distribution. The system frequency requirements from Section II-A are specified in two parts to reflect these two regimes.

Analysis of data collected from one location in the Nordic power system [32] shows frequency deviations are less likely than predicted by a Gaussian distribution up to $f_{o}-3 \sigma$, where $\sigma$ is the standard deviation of frequency measurements. The Gaussian regime ends before frequency values of $f_{o}-6 \sigma$, which are expected to occur once every 2000 years in a Gaussian distribution, but were observed 6 times in one year.

Within the Gaussian regime of frequency measurements, the Gauss error function (erf) gives a closed form solution to eq. (6) and eq. (7) when the frequency standard deviation $\sigma$ is known. The normal distribution is symmetric around the mean value, so we can define:

$$
\Delta f^{\prime}=\Delta f_{\min }^{\prime}=\Delta f_{\max }^{\prime} .
$$

Eq. (6) and eq. (7) can then be combined and solved for $\Delta f^{\prime}$ :

$$
\operatorname{erf}\left(\frac{\Delta f^{\prime}}{\sigma \sqrt{2}}\right)=P_{\text {ok }} .
$$

A narrow system frequency PDF is desirable when using system frequency as an information carrier. Without increasing the power and speed of frequency regulation resources, the frequency PDF seen by FS-DER can be narrowed by lengthening the low pass filter shown in Fig. 4. The cost of a longer low pass filter is an increased $t_{\text {hold }}$ to allow the filtered frequency values $(\hat{f})$ to converge to the system average frequency.

\section{DISPATCH OF FS-DER}

By relaxing the operational constraint that the frequency setpoint must always be the nominal frequency $\left(f_{\mathrm{s}} \neq f_{\mathrm{o}}\right)$ during normal operation, system frequency can be modulated to communicate the value of a single discrete variable to FSDER. In systems with low $R O C O F_{\mathrm{pfc}}$, the time to transition between setpoints $t_{\mathrm{ch}}$ will be large, therefore it is only feasible to encode one symbol per time period, and decoding must be time-invariant. A further restriction with this protocol is that state changes only occur between adjacent states, as shown in Fig. 6. Finally, the design assumes the hold time $t_{\text {hold }}$ is long enough to give a high probability of the sampled and filtered frequency $\hat{f}$ falling within the decision region of the intended state.

Both the transmitter and receiver need to agree $a$ priori on frequency setpoint and threshold values. Frequency thresholds 


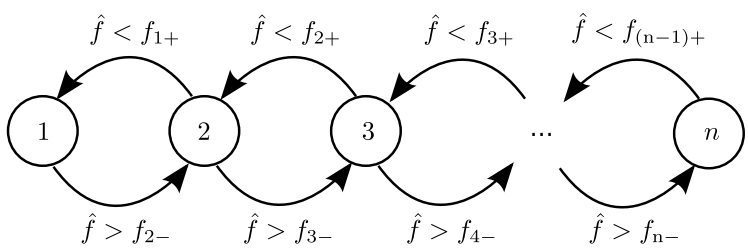

Figure 6. When dispatching FS-DER by modulating system frequency, state transitions only occur between neighboring states.

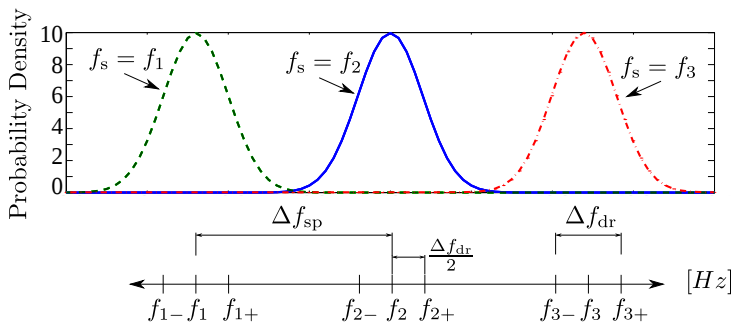

Figure 7. Top: Probability density of frequency samples for three system frequency setpoints: $f_{1}, f_{2}, f_{3}$. Below: The placement of set-points and the threshold values for dispatch of discrete blocks of FS-DER.

must be chosen so that they are separated by enough distance to avoid spurious unintended state changes during $t_{\text {hold }}$. A wide $\Delta f_{\mathrm{sp}}$ avoids spurious changes to FS-DER state, but a wide $\Delta f_{\mathrm{sp}}$ simultaneously reduces the number of symbols feasible within in a given bandwidth and increases $t_{\mathrm{ch}}$.

Assume the size of the decision region $\Delta f_{\mathrm{dr}}[\mathrm{Hz}]$ to be the same for all symbols and defined as:

$$
\Delta f_{\mathrm{dr}}=f_{S+}-f_{S-} .
$$

To find appropriate frequency thresholds for encoding system states, the designer needs to be given a goal for the desired probability of a FS-DER residing in an unintended state $\left(P_{\mathrm{e}}\right)$, and the PDF of system frequency measurements. The minimum distance between a frequency setpoint $f_{S}$, and the neighboring setpoints $f_{(S-1)}, f_{(S+1)}$ is found by first finding a frequency interval $\left[f_{\mathrm{sp}-}^{\prime}, f_{\mathrm{sp}+}^{\prime}\right]$ such that:

$$
\mathrm{P}\left(f \in\left[f_{\mathrm{sp}-}^{\prime}, f_{\mathrm{sp}+}^{\prime}\right] ; f_{\mathrm{o}}\right)=1-P_{\mathrm{e}} .
$$

Then $\Delta f_{\mathrm{sp}}$ is given by:

$$
\Delta f_{\mathrm{sp}}=\frac{1}{2}\left(f_{\mathrm{sp}+}^{\prime}-f_{\mathrm{sp}-}^{\prime}+\Delta f_{\mathrm{dr}}\right) .
$$

Fig. 7 shows the placement of set-points and state change thresholds for a system with 3 symbols, and a Gaussian PDF for frequency measurements. In this case, value of $\Delta f_{\mathrm{sp}+}$ and $\Delta f_{\text {sp- }}$ can be found using the Gauss error function as in eq. (15):

$$
\begin{gathered}
f_{\mathrm{sp}}^{\prime}=f_{\mathrm{sp}-}^{\prime}=f_{\mathrm{sp}+}^{\prime} \\
\operatorname{erf}\left(\frac{f_{\mathrm{sp}}^{\prime}}{\sigma \sqrt{2}}\right)=1-P_{\mathrm{e}} .
\end{gathered}
$$

For a given frequency bandwidth $f_{\mathrm{w}}$, the number of discrete symbols $N$ encoded in that bandwidth is one more than the

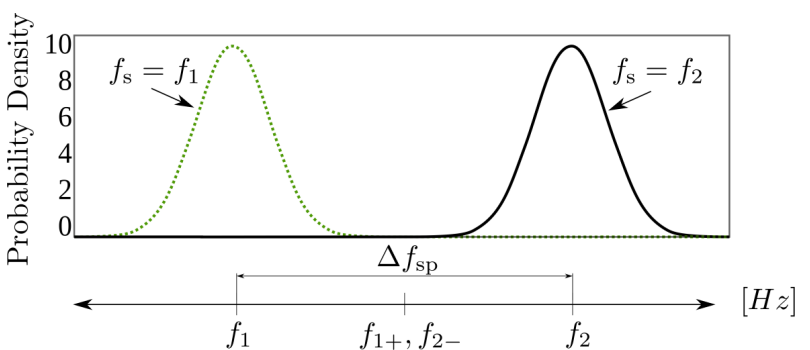

Figure 8. Top: Probability density of frequency samples for two system frequency setpoints: $f_{1}, f_{2}$. Below: The placement of set-points and the threshold values for PFC where $f_{1+}=f_{2-}$.

number of whole frequency spacings that fit in the bandwidth:

$$
N=1+\left\lfloor\frac{f_{\mathrm{w}}}{\Delta f_{\mathrm{sp}}}\right\rfloor .
$$

\section{Generator Speed Governors as Transmitters FOR POWER FREQUENCY COMMUNICATION}

In synchronous areas that allow a high $R O C O F_{\mathrm{pfc}}$, system frequency can be modulated fast enough to allow arbitrary digital information to be transmitted to FS-DER. In contrast to the protocol described in section $\mathrm{V}$ which transmitted the value of a single variable, this protocol can transmit the values of many variables allowing the operator to distribute more information about the system state.

The transmitter (generator) and receiver (FS-DER) must be synchronized, so a data clock is recovered and data packages delimited. Synchronization of clocks and package delimiters is done with a preamble, as in TWACS [2], and not elaborated upon. The decoding of frequency measurements is time independent relative to the start of the data package.

The placement of frequency setpoints for PFC uses the eq. (17) where the decision region is chosen to be $\Delta f_{\mathrm{dr}}=\Delta f_{\mathrm{sp}}$, so there is no hysteresis region between adjacent symbols, as shown in Fig. 8. This wider decision region maps each frequency sample to the nearest setpoint value. The frequency samples taken during $t_{\mathrm{ch}}$ will be ignored, and only frequency samples taken during $t_{\text {hold }}$ are decoded.

When the frequency of the data clock is below twice the AC system frequency $\left(t_{\mathrm{clk}}^{-1} \leq 2 f_{\mathrm{o}}\right)$, dedicating cycles to rise/fall transition between frequencies is unnecessary $\left(t_{\mathrm{ch}}=0\right)$. The transmitter is able to independently regulate the time between zero-crossings of each half-cycle and thus achieves the highest possible data transmission rate of PFC.

Higher level protocols can build upon the physical layer provided by PFC, but the relatively low bandwidth of PFC will force application designers to consider the tradeoff between the length of data transmitted and the time taken for transmission.

\section{Case Studies on Islanded Power Systems}

Using system frequency to encode information is most relevant for small synchronous systems because they already have a relatively wide range for their system frequency, and their generally smaller capacity margins motivate the use of energy management systems. 
Table I

SUGGESTED PARAMETER VALUES FOR FREQUENCY SET-POINTS ON CHRISTIANS $\varnothing$ WITH 7 SYMBOLS, SYMMETRIC AROUND $f_{\mathrm{O}}$.

\begin{tabular}{|l|l|l|}
\hline Parameter & Relative Value & Value \\
\hline$f_{o}$ & & $50 \mathrm{~Hz}$ \\
$\Delta f_{\mathrm{sp}}$ & & $275 \mathrm{mHz}$ \\
$\Delta f_{\mathrm{dr}}$ & $2 \sigma$ & $100 \mathrm{mHz}$ \\
\hline$f_{1}$ & $f_{2}-\Delta f_{\mathrm{sp}}$ & $49.175 \mathrm{~Hz}$ \\
$f_{2}$ & $f_{3}-\Delta f_{\mathrm{sp}}$ & $49.450 \mathrm{~Hz}$ \\
$f_{3}$ & $f_{\mathrm{o}}-\Delta f_{\mathrm{sp}}$ & $49.725 \mathrm{~Hz}$ \\
$f_{4}$ & $f_{\mathrm{o}}$ & $50.000 \mathrm{~Hz}$ \\
$f_{5}$ & $f_{\mathrm{o}}+\Delta f_{\mathrm{sp}}$ & $50.275 \mathrm{~Hz}$ \\
$f_{6}$ & $f_{5}+\Delta f_{\mathrm{sp}}$ & $50.550 \mathrm{~Hz}$ \\
$f_{7}$ & $f_{6}+\Delta f_{\mathrm{sp}}$ & $50.825 \mathrm{~Hz}$ \\
\hline
\end{tabular}

The small synchronous system studied is a microgrid located on the island of Christians $\varnothing$ in Denmark, a decommissioned naval base which is a popular tourist destination. Around 100 people live permanently on the $0.22 \mathrm{~km}^{2}$ island and their electric power generation fleet is composed of 4 diesel fueled generators. The analysis that follows assumes that the operator will live up to the frequency quality requirements of small islands presented in Section II-A.

\section{A. Christians $\phi$ : Diesel Powered Micro-Grid}

Two bottle cooling refrigerators with FS-DER functionality have been installed as part of a field experiment [27]. Frequency measurements were collected over a period of 4 months [33]. The timeseries of frequency measurements had a standard deviation of $\sigma=62 \mathrm{mHz}$. Passing the frequency measurements through a 5-minute moving average filter reduced the standard deviation to $\sigma=50 \mathrm{mHz}$.

The frequency distribution of Christians $\emptyset$ was centered around $50.12 \mathrm{~Hz}$, so taking this as $f_{\mathrm{o}}$, the PDF is:

$$
\mathrm{P}\left(f \in\left[f_{\mathrm{o}}-130 \mathrm{mHz}, f_{\mathrm{o}}+110 \mathrm{mHz}\right] ; f_{\mathrm{o}}\right)=1-0.05 .
$$

Assuming that $f_{\mathrm{o}}$ is a setpoint value that can be arbitrarily changed, and assuming that the lower absolute frequency limit $f_{\text {limit- }}$ and upper absolute frequency limit $f_{\text {limit+ }}$ are not breached during credible contingencies, the range of valid setpoints given by eq. (9) is [49.13 Hz, $50.89 \mathrm{~Hz}]$.

Turning to the spacing between frequency setpoints $\Delta f_{\mathrm{sp}}$, we take the desired error rate to be $P_{\mathrm{e}}=10^{-4}$. The decoder operates with a 5-minute moving average low pass filter. The detection region is given as $\Delta f_{\mathrm{dr}}=100 \mathrm{mHz}$. Using eq. (17), analysis of the filtered data reveals $f_{\text {sp- }}^{\prime}=49.83 \mathrm{~Hz}$ and $f_{\mathrm{sp}+}^{\prime}=50.28 \mathrm{~Hz}$, giving $\Delta f_{\mathrm{sp}}=275 \mathrm{mHz}$ by eq. (18). By eq. (21), the number of distinct symbols feasible in this system is 7. The allocation of frequency setpoints is shown in table I with $f_{\mathrm{o}}$ re-calibrated to the standard $50 \mathrm{~Hz}$.

The length of the low pass filter ( $5 \mathrm{~min}$.) gives a minimum $t_{\text {hold }}=300 \mathrm{~s}$. Even if we generously assume $t_{\mathrm{ch}}=0$, the best case baud rate is 12 symbols per hour.

The frequency setpoints for PFC must be separated by $\Delta f_{\mathrm{sp}}=450 \mathrm{mHz}$. The feasible data rate of PFC is also 12 bits per hour because increased spacing between frequency setpoints does not significantly influence $t_{\mathrm{ch}}$.

\section{B. Laboratory Test: Inverter Dominated Micro-Grid}

The increasing cost of diesel fuel, emissions constraints, and decreasing costs of RES have motivated the operator on Christians $\varnothing$ to investigate replacing their current generation fleet with RES which may be inverter connected. To simulate the energy source in such an system, a VSI in our laboratory was tested to characterize the speed of response to changes in frequency setpoints. The inverter, produced by IE Power, had a rated DC input of $136 \mathrm{~V}$, and rated 3-phase $\mathrm{AC}$ output of $400 \mathrm{~V}$, a power rating of $20 \mathrm{kVA}$, and a frequency range of $47.5 \mathrm{~Hz}$ to $52.5 \mathrm{~Hz}$. The DC side of the inverter was fed by a vanadium flow battery whose performance was characterized in [34]. Frequency setpoints are communicated to the inverter by means of a current loop, an inherently analog control signal. Instead of directly measuring this current, the voltage across the input terminals of the inverter was measured, as the measured voltage is proportional to the current flowing in the circuit. The input voltage indicating the frequency setpoint and the output voltage of one phase of the inverter with noload was measured by a Yokogawa WT500 power analyzer and a Yokogawa DL750 oscilloscope. The power analyzer measured voltage at a relatively high resolution $(1 \mathrm{mV})$, but a relatively low temporal resolution $(100 \mathrm{~ms})$. The oscilloscope had a high temporal resolution (100 $\mu \mathrm{s})$, but lower voltage resolution $(33 \mathrm{mV})$. Separate test runs of the same frequency setpoint sequence were recorded using the power analyzer and oscilloscope.

The power analyzer calculated AC frequency at each time step using an undisclosed internal algorithm. AC frequency was extracted from the oscilloscope data using a zero-crossing algorithm with linear interpolation, a method validated in a previous experiment with FS-DER [27]. Measurement noise found in the $100 \mu$ s data series was mitigated by applying at 4-sample sliding-window moving average, and the cycleby-cycle frequency calculations were again averaged over two periods to reduce variance. Assuming that an analog-to-digital converter similar to the oscilloscope is feasible to implement in an embedded system, the frequency measurements derived from analysis of oscilloscope data closely matches what would be seen by a low-cost FS-DER.

Steady-state frequency measurements taken by the power analyzer showed a standard deviation of $3.5 \mathrm{mHz}$ and

$$
\mathrm{P}\left(f \in\left[f_{\mathrm{o}}-14 \mathrm{mHz}, f_{\mathrm{o}}+14 \mathrm{mHz}\right] ; f_{\mathrm{o}}\right)=1-10^{-4}
$$

and

$$
\mathrm{P}\left(f \in\left[f_{\mathrm{o}}-8 \mathrm{mHz}, f_{\mathrm{o}}+8 \mathrm{mHz}\right] ; f_{\mathrm{o}}\right)=1-0.05 .
$$

A sequence of frequency setpoints was programmed using a high level software API, and these software commands were converted into current levels by a digital-to-analog converter. Measuring the frequency setpoint at the terminals of the inverter removed any delays introduced by the high-level software, and only the timing of the inverter is tested. Changes to frequency setpoints are tested at 3 orders of magnitude: $\pm 1 \mathrm{~Hz}, \pm 100 \mathrm{mHz}$, and $\pm 10 \mathrm{mHz}$, shown in Fig. 9 .

Fig. 10 shows a close up as the inverter changes frequency setpoint from $51.00 \mathrm{~Hz}$ to $49.00 \mathrm{~Hz}$, using data from the 


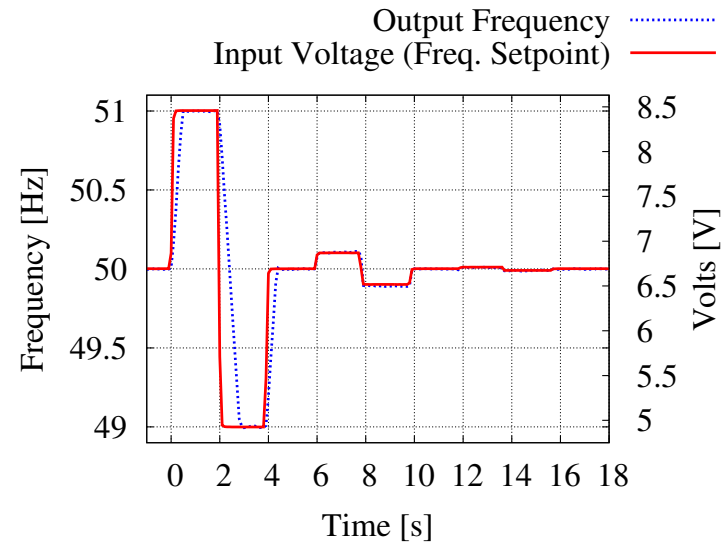

Figure 9. Test sequence of frequency setpoints given to inverter, and the output frequency as measured by the power analyzer. At time $0 \mathrm{~s}$ the setpoint changes from $50 \mathrm{~Hz}$ to $51 \mathrm{~Hz}$. After 2 seconds, the setpoint is changed to 49 $\mathrm{Hz}$, and at time $4 \mathrm{~s}$ the setpoint returns to $50 \mathrm{~Hz}$. At time $6 \mathrm{~s}$ the setpoint is changed to $50.10 \mathrm{~Hz}$, at time $8 \mathrm{~s}$ it is changed to $49.90 \mathrm{~Hz}$. Finally, barely visible at this scale, at time $12 \mathrm{~s}$ the setpoint is changed to $50.01 \mathrm{~Hz}$, then $49.99 \mathrm{~Hz}$ at time 14 .

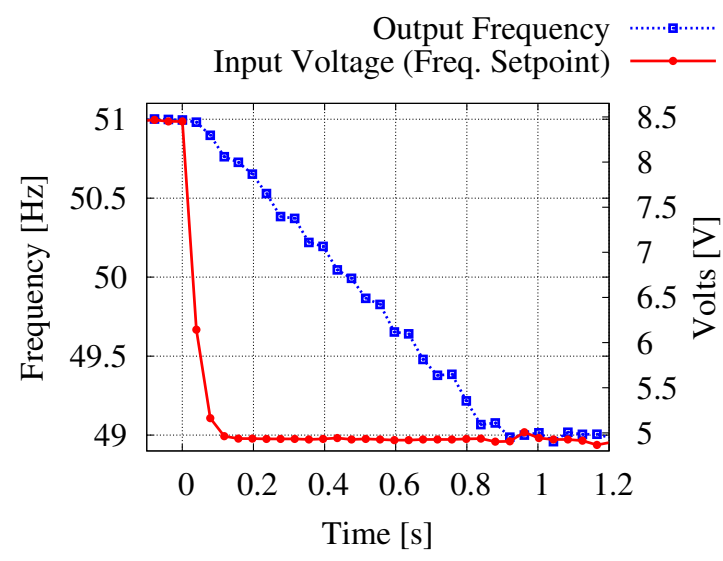

Figure 10. Step change of frequency setpoint of $-2 \mathrm{~Hz}$. Voltage and frequency measurements derived from the oscilloscope data points are shown at $40 \mathrm{~ms}$ (2-cycle) intervals.

oscilloscope. At time 0 , the voltage across the inverter input terminals falls from $8.5 \mathrm{~V}$ to $5 \mathrm{~V}$. The output frequency immediately begins to decline, and stabilizes $900 \mathrm{~ms}$ after the input began to change. A change of $2 \mathrm{~Hz}$ in $0.9 \mathrm{~s}$ gives a ROCOF of $2.2 \mathrm{~Hz} / \mathrm{s}$.

Fig. 11 shows the response to $-200 \mathrm{mHz}$ change in frequency setpoint starting at $50.10 \mathrm{~Hz}$, again using data from the oscilloscope. At time 0 the input signal begins to change, and $140 \mathrm{~ms}$ later, output frequency has dropped below the intended setpoint of $49.90 \mathrm{~Hz}$, giving a ROCOF of $1.4 \mathrm{~Hz} / \mathrm{s}$.

Finally, the response of the inverter to a $-20 \mathrm{mHz}$ frequency setpoint change is shown in Fig. 12. This figure is based on data from the power analyzer because the change in input voltage was too small to measure with the oscilloscope. The output frequency falls below the setpoint value one time step $(100 \mathrm{~ms})$ after the input begins to change. The time resolution of the power analyzer frequency measurements was insufficient to determine if the output frequency changed faster than the observed $0.2 \mathrm{~Hz} / \mathrm{s}$.

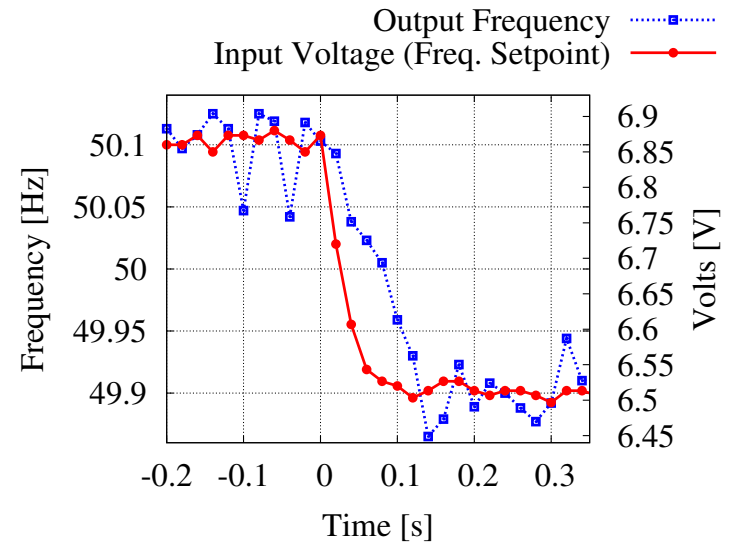

Figure 11. Step change of frequency setpoint of $-200 \mathrm{mHz}$. Voltage and frequency measurements derived from the oscilloscope data points are shown at $20 \mathrm{~ms}$ (1-cycle) intervals.

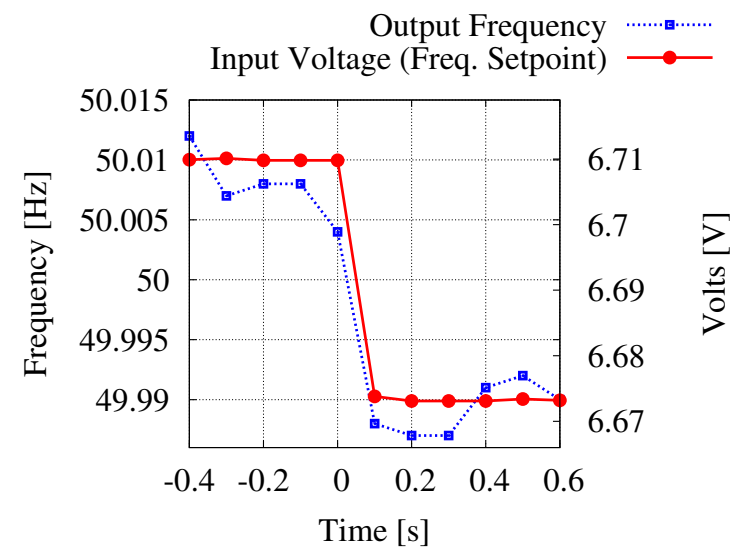

Figure 12. Step change of frequency setpoint of $-20 \mathrm{mHz}$. Voltage and frequency measurements derived from the power analyzer with data points shown at $100 \mathrm{~ms}$ (5-cycle) intervals.

To find the range of feasible frequency setpoint values we use eq. (14) and eq. (24), to specify the value of $\Delta f^{\prime}=8$ $\mathrm{mHz}$. This results in an available bandwidth of $f_{\mathrm{w}}=1.98 \mathrm{~Hz}$.

To find the spacing between frequency setpoints $\Delta f_{\mathrm{sp}}$, we take the desired error rate to be $P_{\mathrm{e}}=10^{-4}$, the same as in Section VII-A. The decision region is given as $\Delta f_{\mathrm{dr}}=2 \sigma=$ $7 \mathrm{mHz}$. Using the definition of $f_{\mathrm{sp}}^{\prime}$ from eq. (19) and the value of $f_{\mathrm{sp}}^{\prime}=14 \mathrm{mHz}$ from eq. (23), eq. (18) gives $\Delta f_{\mathrm{sp}}=17.5$ $\mathrm{mHz}$.

From eq. (21) we see that the bandwidth can accommodate up to 114 discrete symbols.

Recall for PFC $\Delta f_{\mathrm{dr}}=\Delta f_{\mathrm{sp}}$, and $f_{\mathrm{sp}}^{\prime}=14 \mathrm{mHz}$, so eq. (18) gives $\Delta f_{\mathrm{sp}}=28 \mathrm{mHz}$. If the $R O C O F_{p f c}$ value of 1.4 $\mathrm{Hz} / \mathrm{s}$ can also be achieved for this smaller change in frequency setpoint, $t_{\mathrm{ch}}=20 \mathrm{~ms}$, or exactly one AC cycle at $50 \mathrm{~Hz}$. The time $t_{\text {hold }}$ is determined by the length of a low-pass filter in the receiver. The zero-crossing algorithm used in this analysis required a low-pass filter of 2-cycles to reduce variance, so if $t_{\text {hold }}=40 \mathrm{~ms}, t_{\mathrm{clk}}=60 \mathrm{~ms}$ giving a maximum bit rate of 16.6 bps. 


\section{DISCUSSION}

Compared to other power line communication protocols, the proposed protocols should be inexpensive to implement because they use existing generator speed governors to encode data and allow decoding devices to be implemented with commodity low-cost microcontrollers. The use of carrier frequencies below the system frequency allows the signal to propagate for an effectively unlimited distance.

Parameter values for the proposed AC frequency modulation system will vary greatly between synchronous systems. Large synchronous systems will have very low $R O C O F_{\text {plc }}$ values because of high inertia. This makes it infeasible to use generator speed governors for PFC, but this does not exclude using the limited available bandwidth for dispatching FS-DER. Inverter dominated synchronous areas have high $R O C O F_{\mathrm{plc}}$ values that enable PFC to be implemented. In the VSI tested in Section VII-B the ROCOF was a nonlinear function of the size of the frequency step, so for this device the denominator in eq. (13) will be a function of $\Delta f_{\mathrm{sp}}$. The maximum ROCOF of the VSI was limited by a parameter in its embedded software, it is possible that the power electronics hardware could support higher ROCOF values.

Detailed discussion of applications of the proposed protocols is outside the scope of this paper, but to illustrate the difference between the two protocols, their application to price dissemination is considered. Using the FS-DER dispatch protocol allows the current electricity price to be reflected by the system frequency, allowing marginal consumers to judge if the utility of their consumption exceeds the current price. With PFC and a bit rate high enough to send the price values for the current and future operation periods, consumers would have the information needed to optimize their consumption across a longer time horizon.

The probability of communication errors, $P_{\mathrm{e}}$, is a design parameter. In practical systems it will not be low enough to entirely eliminate the possibility of disturbances causing errors in communication. The applications that utilize this communication system will need to be robust in the presence of such errors. For example, when using PFC, checksums in the protocol can be employed to validate the integrity of messages. When using system frequency to dispatch FS-DER by synchronous generators, the linkage between low frequency and excess load requires that lower frequencies lead to lower system load and/or greater DG production.

Many loads are indifferent to variations in system frequency, but off-nominal frequency may lead to higher losses in machines optimized for a particular frequency, such as generators and legacy industrial processes [35]. This concept could have a negative effect on machine efficiency; however, there will be systems where the the benefit of distributing information for demand response outweighs these costs.

\section{CONCLUSION}

The paper has shown how existing frequency regulation resources can be applied to transmitting discrete information. Explicitly signaling discrete system states by generating offnominal system frequency values can be used as a novel narrowband unidirectional broadcast communications channel between system operators and FS-DER. Systems that are restricted to slow changes in system frequency can communicate the value of a single discrete variable to FS-DER, while systems that allow fast changes to system frequency can implement general purpose unidirectional broadcast power frequency communication (PFC). Using generators as PFC transmitters does not require any additional power electronics and eliminates the energy losses associated with previous PFC transmitters.

Data collected from an diesel fueled islanded power system shows that it is feasible to encode 7 discrete symbols for FSDER dispatch. Laboratory tests of at $20 \mathrm{~kW}$ VSI showed that it was able to respond to frequency setpoint changes with a ROCOF of between 2.2 and $0.2 \mathrm{~Hz} / \mathrm{s}$ depending on the size of the setpoint change. In a VSI dominated micro-grid, analysis of the experimental data shows that a PFC bit rate of $16.6 \mathrm{bps}$ is feasible.

\section{REFERENCES}

[1] F. Schweppe, R. Tabors, J. Kirtley, H. Outhred, F. Pickel, and A. Cox, "Homeostatic utility control," Power Apparatus and Systems, IEEE Transactions on, vol. PAS-99, no. 3, pp. $1151-1163$, may 1980.

[2] S. Mak and D. Reed, "TWACS, A new viable two-way automatic communication system for distribution networks. Part I: Outbound communication," Power Apparatus and Systems, IEEE Transactions on, vol. PAS-101, no. 8, pp. $2941-2949$, aug. 1982.

[3] S. T. Mak, "A new method of generating TWACS type outbound signals for communication on power distribution networks," Power Apparatus and Systems, IEEE Transactions on, vol. PAS-103, no. 8, pp. $2134-$ 2140, aug. 1984.

[4] P. J. Douglass, S. You, and K. Heussen, "Broadcast communication by system frequency modulation," in Smart Grid Communications (SmartGridComm), 2012 IEEE Third International Conference on, 2012, pp. 199-204.

[5] J. R. Pillai, K. Heussen, and P. A. Østergaard, "Comparative analysis of hourly and dynamic power balancing models for validating future energy scenarios," Energy, vol. 36, no. 5, pp. 3233 - 3243, 2011.

[6] CENELEC, "EN 50160: Voltage characteristics of electricity supplied by public electricity networks," 2010.

[7] ENTSO-E, "Draft Network Code for Requirements for Grid Connection applicable to all Generators," 24 Jan. 2012.

[8] P. Kundur, N. Balu, and M. Lauby, Power system stability and control. McGraw-Hill New York, 1994.

[9] V. Terzija, "Adaptive underfrequency load shedding based on the magnitude of the disturbance estimation," Power Systems, IEEE Transactions on, vol. 21 , no. 3, pp. $1260-1266$, aug. 2006.

[10] IEEE Task Force, "Standard load models for power flow and dynamic performance simulation," Power Systems, IEEE Transactions on, vol. 10, no. 3, pp. $1302-1313$, aug 1995.

[11] J. Undrill, "Power and Frequency Control as it Relates to Wind-Powered Generation," Ernest Orlando Lawrence Berkeley National Laboratory, Tech. Rep. LBNL-4143E, Oct. 2010.

[12] B. Parsons, M. Milligan, B. Zavadil, D. Brooks, B. Kirby, K. Dragoon, and J. Caldwell, "Grid impacts of wind power: a summary of recent studies in the united states," Wind Energy, vol. 7, no. 2, pp. 87-108, 2004.

[13] I. Egido, F. Fernandez-Bernal, P. Centeno, and L. Rouco, "Maximum frequency deviation calculation in small isolated power systems," Power Systems, IEEE Transactions on, vol. 24, no. 4, pp. $1731-1738$, nov. 2009.

[14] J. Vieira, W. Freitas, W. Xu, and A. Morelato, "Efficient coordination of rocof and frequency relays for distributed generation protection by using the application region," Power Delivery, IEEE Transactions on, vol. 21 , no. 4 , pp. $1878-1884$, oct. 2006.

[15] "Rate of Change of Frequency (ROCOF) DS3 Advisory Council Discussion Paper," Online, EirGrid, SONI, Tech. Rep., 2 Feb. 2012, accessed September 19, 2012. [Online]. Available: http://www.eirgrid.com/operations/ds3 
[16] UCTE, "Operations Handbook Appendix 1: Load Frequency Control," 16 Jun. 2004

[17] L. A. C. Lopes, F. Katiraei, K. Mauch, M. Vandenbergh, and L. Arribas, "PV Hybrid Mini-Grids: Applicable Control Methods for Various Situations," International Energy Agency, Tech. Rep., Apr. 2012.

[18] M. Reza, A. Dominguez, P. Schavemaker, A. Asmara, F. Viawan, and W. Kling, "Controlling the power balance in an 'empty network'," Int. J. of Energy Technology and Policy, vol. 5, no. 5, pp. $584-603,2007$.

[19] J. Short, D. Infield, and L. Freris, "Stabilization of grid frequency through dynamic demand control," Power Systems, IEEE Transactions on, vol. 22, no. 3, pp. $1284-1293$, aug. 2007.

[20] P. J. Douglass, R. Garcia-Valle, P. Nyeng, J. Østergaard, and M. Togeby, "Smart demand for frequency regulation: Experimental results," Smart Grid, IEEE Transactions on, vol. 4, no. 3, pp. 1713-1720, 2013.

[21] M. Donnelly, D. Trudnowski, S. Mattix, and J. Dagle, "Autonomous Demand Response for Primary Frequency Regulation," Pacific Northwest National Laboratory, Tech. Rep., Jan. 2012.

[22] ENTSO-E, "Draft Demand Connection Codes," 27 Jun. 2012.

[23] Zhao Xu, J. Østergaard, and M. Togeby, "Demand as frequency controlled reserve," Power Systems, IEEE Transactions on, vol. 26, no. 3, pp. $1062-1071$, Aug. 2011.

[24] CENELEC, "EN 50065-1: Signalling on low-voltage electrical installations in the frequency range $3 \mathrm{kHz}$ to $148,5 \mathrm{kHz}$ - Part 1: General requirements, frequency bands and electromagnetic disturbances," 2011.

[25] S. Galli, A. Scaglione, and Z. Wang, "For the grid and through the grid: The role of power line communications in the smart grid," Proceedings of the IEEE, vol. 99, no. 6, pp. $998-1027$, june 2011.

[26] "Aclaratech," Online, 2012, accessed September 27, 2012. [Online]. Available: http://www.aclaratech.com/

[27] P. Douglass, R. Garcia-Valle, P. Nyeng, J. Østergaard, and M. Togeby, "Demand as Frequency Controlled Reserve: Implementation and practical demonstration," in Innovative Smart Grid Technologies (ISGT Europe), 2011 2nd IEEE PES International Conference and Exhibition on, 2011, pp. 1-7.

[28] VDE, "VDE-AR-N 4105:2011-08 Power generation systems connected to the low-voltage distribution network - Technical minimum requirements for the connection to and parallel operation with low-voltage distribution networks," 1 Aug. 2010. [Online]. Available: http://www.vde.com/en/fnn/pages/n4105.aspx

[29] SMA Technogie AG, "Sunny Island 5048u Installation and Instruction Manual, Version 1.0," 2007.

[30] P.-O. Moix, "A Minigrid of Individual Solar Home Systems," in 6th European Conference of PV-Hybrids and Mini-Grids, 2012.

[31] B. Biegel, L. H. Hansen, P. Andersen, and J. Stoustrup, "Primary Control by ON/OFF Demand-Side Devices," IEEE Transactions on Smart Grid, preprint.

[32] Z. Xu, J. Ostergaard, M. Togeby, and F. Isleifsson, "Evaluating frequency quality of nordic system using pmu data," in Power and Energy Society General Meeting - Conversion and Delivery of Electrical Energy in the 21st Century, 2008 IEEE, 2008, pp. 1-5.

[33] P. J. Douglass, "Integrating Autonomous Load Controllers in Power Systems," Ph.D. dissertation, Technical University of Denmark, Dec. 2013.

[34] H. Bindner, C. Krog Ekman, O. Gehrke, and F. Isleifsson, Characterization of Vanadium Flow Battery, ser. Denmark. Forskningscenter Risoe. Risoe-R. Danmarks Tekniske Universitet, Ris $\varnothing$ Nationallaboratoriet for Bæredygtig Energi, 2010.

[35] ENTSO-E, "Supporting Paper for the Load-Frequency and Reserves Network Code - Working Draft," 23 Jan. 2013. 